\title{
Text Generation for Intermediate Non-native Speakers of English
}

\author{
Xinyu Deng ${ }^{\dagger}$ and Jun-ichi Nakamura
}

\begin{abstract}
This paper describes the microplanner of the SILK system which can generate texts appropriate for intermediate non-native users on discourse level. Four factors (i.e. nucleus position, between-text-span punctuation, embedded discourse markers and punctuation pattern) are regarded to affect the readability at discourse level. It is the preferences among these factors that decide the readability. Since the number of possible combinations of the preferences is huge, we use Genetic Algorithm to solve such a problem. We adopt two methods to evaluate the system: one is evaluating the reliability of the SILK system by analysing how often it re-generates corpus texts, another is judging readability by human subjects. The evaluation results show that the system is reliable and the generation results are appropriate for intermediate non-native speakers on discourse level.
\end{abstract}

Key Words: text generation, discourse level, Genetic Algorithm, intermediate non-native speakers of English

\section{Background}

At present, the population of non-native speakers is twice that of native speakers of English. In natural language generation (NLG), although some text generation systems (McKeown 1985; Goldberg, Driedger, and Kittredge 1994; Bateman 1997) had been developed, their target users were native speakers. Since the population of non-native speakers is huge, text generation for non-native speakers whose English level is lower is a promising application area. At present, we are developing a text generation system which is called SILK (text generation System for Intermediate Level non-native speaKers ${ }^{1}$ ). This paper describes the microplanner of the SILK system. The domain of the generated texts is natural and pure science.

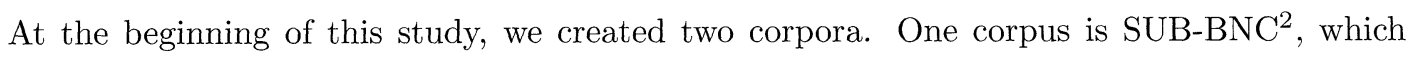
contains texts whose target audience was native speakers. Another is TANN, which contains texts

\footnotetext{
$\dagger$ Kyoto University, Graduate School of Informatics

1 Generally speaking, non-native speakers are divided into three levels: primary (middle school level), intermediate (high school level) and advanced (university level). The users of this study are assumed to be at intermediate level.

2 SUB-BNC is a sub-corpus of British National Corpus. See http://www.natcorp.ox.ac.uk/
} 
whose target audience was intermediate non-native speakers ${ }^{3}$. The two corpora are comparable, because their size (200,000 words each), domain (natural and pure science) and medium (book) are consistent with each other. The results of corpus analysis show that the usage of some words is different between the two corpora. For example, within SUB-BNC, $48 \%$ of subordinate clauses beginning with if occur in the first span, while within TANN, about $80 \%$ occur in the first span. Within SUB-BNC, $20 \%$ of subordinate clauses beginning with although occur in the first span, while within TANN, 70\% occur in the first span. Moreover, for the subordinate clauses beginning with because, within SUB-BNC, $8 \%$ occur in the first span, while within TANN, $29 \%$ occur in the first span. Therefore, we realized that although some text generation systems had been developed for native speakers, we ought to explore the generation strategies for non-native users independently without taking into account the case of native speakers.

In this study, four factors (i.e. nucleus position, between-text-span punctuation, embedded discourse markers and punctuation pattern) are regarded to affect the readability on discourse level. We claim that it is the preferences among these factors rather than the factors themselves that decide the readability. That is, capturing these preferences properly can generate a text which is appropriate for intermediate non-native speakers on discourse level. We use Genetic Algorithm ${ }^{4}$ to realize this consideration. The rest of the paper is arranged as follows. Section 2 describes the microplanner. Section 3 introduces the preferences among the four factors. Section 4 illustrates how to generate texts by Genetic Algorithm. Section 5 describes the evaluation methods. Section 6 introduces the related work. In Section 7, we draw a conclusion.

\section{An introduction to the microplanner}

This study focuses on the microplanner of the SILK system. The task of the microplanner is to transform text representations from hierarchical tree structures into ordered individual sentences. In other words, the microplanner decides how the sentences from the tree will be ordered and punctuated, and selects one text appropriate for intermediate non-native users.

The input of the microplanner is the text structure represented by Rhetorical Structure Theory (RST) (Mann and Thompson 1988), for example, the RST-tree shown in Fig. 1. In an RST-tree, non-terminal nodes represent discourse relations, while terminal nodes represent sentences. In Fig. 1, Node 0 represents "elaboration" relation, and Node 1 represents sentence "plant at the

\footnotetext{
3 The texts within TANN were selected from the high school students' textbooks published in China and in Japan.

4 The framework of Simple Genetic Algorithm used in this study was obtained from (Hirano 2003).
} 


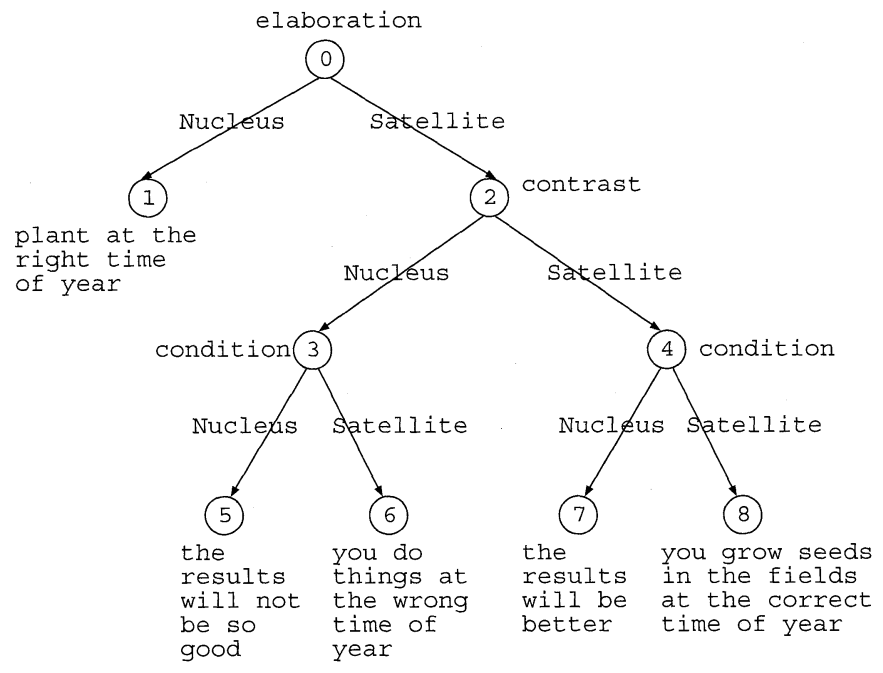

Fig. 1 Input of the microplanner

Plant at the right time of year. If you grow seeds in the fields at the correct time of year, the results will be better. But if you do things at the wrong time of year, the results will not be so good.

Fig. 2 Output of the microplanner

right time of year". We have the following two reasons to use RST.

(1) RST is suitable to represent the discourse structure of any genre of texts. Therefore, there is no problem to use RST to represent the structure of the text whose domain is natural and pure science.

(2) In RST, the discourse relations initially defined is an open set. That is, the researchers can add or modify relations according to their needs. In this study, we defined 12 discourse relations: background, condition, contrast, elaboration, evaluation, example, list, purpose, reason, restatement, summary, time.

The output of the microplanner is a coherent text that contains individual sentences which are ordered and punctuated, for example, the text shown in Fig. 2.

\section{Preferences among factors affecting the readability on discourse level}

As mentioned in Section 1, we think that four factors affect the readability on discourse level. In this section, we introduce the preferences among these factors. 
In an RST-tree, each non-terminal node is the root of a sub-tree. We assume that a candidate generation result of each sub-tree has two kinds of structures. One is called local structure, in which the nucleus and the satellite are regarded as nodes. The local structure can be represented as "node + between-text-span punctuation + node". Another is called linear structure, which is linear ordered sentences. For example, in Fig. 1, the sub-tree whose root is Node 3 has two child nodes: Node 5 and Node 6 . If the local structure of a candidate generation result is "Node $6+$ comma + Node 5", the linear structure of this candidate generation result is "If you do things at the wrong time of year, the results will not be so good.".

\subsection{Preferences for nucleus position in the local structure}

In the field of NLG, the usage of discourse markers has been regarded as an important problem (Elhadad and McKeown 1990; Moser and Moore 1995; Eugenio, Moore, and Paolucci 1997). At present, the SILK system can generate six discourse relations, namely, "condition" relation, "contrast" relation, "example" relation, "reason" relation, "elaboration" relation and "list" relation. We use discourse markers if, but, for example and because to signal the first four relations. No discourse marker is used to signal the later two. Moreover, "list" relation only has two child nodes. We explored the preferences for the nucleus position of each relation by using machine learning program C4.5 (see Appendix) and analysing corpus TANN. We divided the nucleus position into the following three states.

(1) Good position. It refers to the position which is the result of machine learning. For example, for "condition" relation signaled by if, the results of machine learning show that nuclei occur in the second span, such as "If you do not go to bed early, you cannot have enough sleep.". Therefore, the second span is the "good position".

(2) Normal position. It refers to a position which is not the result of machine learning but that of corpus analysis. For example, for "condition" relation signaled by if, although machine learning results show that the nuclei occur in the second span, corpus analysis results show that about $20 \%$ of nuclei occur in the first span, such as "In general, intelligence is not connected well with age, especially if a person is healthy and active.". Therefore, the first span is a "normal position".

(3) Bad position. It refers to the position which is neither the result of machine learning nor that of corpus analysis. For example, for "elaboration" relation, nuclei never occur in the second span. Therefore, the second span is a "bad position".

The three states of the nucleus position for six relations are shown in Table 1. 
Table 1 Three states of nucleus position for six discourse relations

\begin{tabular}{|c|c|c|c|}
\hline & \multicolumn{3}{|c|}{ Nucleus position } \\
\hline & Good & Normal & Bad \\
\hline \multirow{2}{*}{$\begin{array}{l}\text { Reason } \\
\text { (because) }\end{array}$} & $\begin{array}{c}\text { second span } \\
\text { (if } \mathrm{R} \text { is "contrast", } \\
\text { "example", or "reason") }\end{array}$ & $\begin{array}{c}\text { first span } \\
\text { (if R is "contrast", } \\
\text { "example" or "reason") }\end{array}$ & \multirow[t]{2}{*}{ none } \\
\hline & $\begin{array}{l}\text { first span } \\
\text { (if R is not "contrast", } \\
\text { "example" or "reason") }\end{array}$ & $\begin{array}{c}\text { second span } \\
\text { (if R is not "contrast", } \\
\text { "example", or "reason") }\end{array}$ & \\
\hline $\begin{array}{c}\text { Contrast } \\
\text { (but) }\end{array}$ & second span & none & first span \\
\hline $\begin{array}{c}\text { Example } \\
\text { (for example) }\end{array}$ & first span & none & second span \\
\hline $\begin{array}{l}\text { Condition } \\
\text { (if) }\end{array}$ & second span & first span & none \\
\hline $\begin{array}{c}\text { Elaboration } \\
\text { (no cue) }\end{array}$ & first span & none & second span \\
\hline $\begin{array}{c}\text { List } \\
\text { (no cue) }\end{array}$ & first span & none & second span \\
\hline
\end{tabular}

Heuristic 1 Preferences among states for nucleus position: good position $>$ normal position $>$ bad position

\subsection{Preferences for between-text-span punctuation in the local struc- ture}

We explore the preferences for between-text-span punctuation in the local structure by using C4.5 and analysing corpus TANN. The method is same as that mentioned in Section 3.1. We divided the between-text-span punctuation into the following three states.

(1) Good punctuation. It refers to the punctuation which is the result of C4.5. For example, for "condition" relation signaled by if, when the nucleus occurs in the second span, the between-text-span punctuation is a comma, such as "If you do not go to bed early, you cannot have enough sleep.". Therefore, comma is the "good punctuation".

(2) Normal punctuation. It refers to a punctuation which is not the result of machine learning 
but that of corpus analysis. For example, for "condition" relation signaled by if, the machine learning results show that when the nucleus occurs in the first span, the betweentext-span punctuation is a space. However, within TANN, the punctuation sometimes is a comma, such as "In general, intelligence is not connected well with age, especially if a person is healthy and active.". Therefore, comma is a "normal punctuation".

(3) Bad punctuation. It refers to the punctuation which is neither the result of machine learning nor that of corpus analysis. For example, for "condition" relation signaled by if, when the nucleus occurs in the second span, a full stop is never used as the betweentext-span punctuation, such as "If you do not go to bed early. You cannot have enough sleep.". Therefore, full stop is a "bad punctuation".

Table 2 Three states of between-text-span punctuation for six discourse relations

\begin{tabular}{|c|c|c|c|c|}
\hline & \multirow{2}{*}{$\begin{array}{l}\text { Nucleus } \\
\text { position }\end{array}$} & \multicolumn{3}{|c|}{ Between-text-span punctuation } \\
\hline & & Good & Normal & Bad \\
\hline \multirow{3}{*}{$\begin{array}{c}\text { Reason } \\
\text { (because) }\end{array}$} & \multirow[t]{2}{*}{ first span } & $\begin{array}{c}\text { space } \\
(\mathrm{Eg} \leq 21)\end{array}$ & $\begin{array}{c}\text { comma } \\
(\mathrm{Eg} \leq 21)\end{array}$ & \multirow[t]{2}{*}{ full stop } \\
\hline & & $\begin{array}{c}\text { comma } \\
(\mathrm{Eg}>21)\end{array}$ & $\begin{array}{c}\text { space } \\
(\mathrm{Eg}>21)\end{array}$ & \\
\hline & second span & comma & none & space, full stop \\
\hline \multirow{3}{*}{$\begin{array}{l}\text { Contrast } \\
\text { (but) }\end{array}$} & first span & none & none & space, comma, full stop \\
\hline & \multirow[t]{2}{*}{ second span } & $\begin{array}{c}\text { comma } \\
(\mathrm{Eg} \leq 29)\end{array}$ & $\begin{array}{l}\text { space, full stop } \\
\qquad(\mathrm{Eg} \leq 29)\end{array}$ & \multirow[t]{2}{*}{ none } \\
\hline & & $\begin{array}{c}\text { full stop } \\
(\mathrm{Eg}>29)\end{array}$ & $\begin{array}{l}\text { space, comma } \\
(\mathrm{Eg}>29)\end{array}$ & \\
\hline \multirow{2}{*}{$\begin{array}{c}\text { Example } \\
\text { (for example) }\end{array}$} & first span & full stop & none & space, comma \\
\hline & second span & none & none & space, comma, full stop \\
\hline \multirow{2}{*}{$\begin{array}{l}\text { Condition } \\
\text { (if) }\end{array}$} & second span & comma & none & space, full stop \\
\hline & first span & space & comma & full stop \\
\hline \multirow{2}{*}{$\begin{array}{c}\text { Elaboration } \\
\text { (no cue) }\end{array}$} & first span & full stop & none & space, comma \\
\hline & second span & none & none & space, comma, full stop \\
\hline \multirow{2}{*}{$\begin{array}{c}\text { List } \\
\text { (no cue) }\end{array}$} & first span & full stop & none & space, comma \\
\hline & second span & none & none & space, comma, full stop \\
\hline
\end{tabular}


The three states of between-text-span punctuation for six relations are shown in Table 2 . The results of machine learning and corpus analysis show that between-text-span punctuation is determined by the nucleus position (see the second column of Table 2).

Heuristic 2 Preferences among states for between-text-span punctuation: good punctuation $>$ normal punctuation $>$ bad punctuation

\subsection{Preferences for embedded discourse markers in the linear structure}

In an embedded structure, two discourse markers are sometimes used to signal two discourse relations. This kind of discourse markers are called embedded discourse markers (EDMs). An embedded structure in which EDMs occur has two discourse markers and three propositions. We divided EDMs into two patterns. Pattern 1 represents the embedded structure in which the first discourse marker immediately precedes the second one and both discourse markers are attached to the second proposition, for example, Text (1). Pattern 2 represents the embedded structure in which discourse markers precede the second and the third proposition respectively, for example, Text (2).

1. You failed the exam. But if you study hard, you can master English.

2. You failed the exam. But you can master English if you study hard.

The research of (Power, Doran, and Scott 1999) explored the problem of how to generate embedded discourse markers from rhetorical structure. However, it discussed the case of native speakers. In (Biber, Johansson, Leech, Conrad, and Finegan 1999), the authors point out that Pattern 1 is more difficult to understand, because placing the subordinate clause in the initial position of the embedded structure can create problems for processing. In (Williams 2003), the author also claims that it is better not use Pattern 1 while generating a text for native speakers. The results of corpus analysis proved their opinion, because within SUB-BNC, the frequency of Pattern 1 and Pattern 2 is 65 and 93. That is, Pattern 2 is more often used. On the contrary, we found that within TANN, the frequency of Pattern 1 and Pattern 2 is 119 and 87. That is, Pattern 1 is more often used. So we can infer that compared with Pattern 2, Pattern 1 is easier to read for intermediate non-native speakers. According to psycholinguistics, one possible explanation is that embedded structures are not easy to understand, so it is necessary to put two discourse markers together to attract the attention of intermediate non-native audiences. Based on the results of corpus analysis, we divided EDMs into the following three states:

(1) Good EDMs. They refer to Pattern 1 of EDMs, such as "but if" and "for example, because".

(2) Normal EDMs. They refer to Pattern 2 of EDMs, such as "for example,... if". 
Table 3 Examples of three states of EDMs

\begin{tabular}{|l|l|l|}
\hline \multicolumn{1}{|c|}{ Good EDMs } & \multicolumn{1}{|c|}{ Normal EDMs } & \multicolumn{1}{c|}{ Bad EDMs } \\
\hline \hline because if & because...if & because but \\
but because & but...because & because for example, \\
but if & but...if & but for example, \\
for example, because & but...for example, & for example, but \\
for example, if & for example,...because & if because \\
\hline
\end{tabular}

(3) Bad EDMs. They refer to the EDMs which could not be found within TANN, such as "for example, but". Actually, if such EDMs were used, the cohesion, naturalness and logicality of the text would be destroyed.

Some examples of the three states of EDMs are shown in Table 3.

Heuristic 3 Preferences among states for EDMs: good EDMs > normal EDMs > bad EDMs

\subsection{Preferences for punctuation pattern in the linear structure}

Punctuation is an indispensable component of written language. During the last decade, researches like (Hovy and Arens 1991; Dale 1991; White 1995; Briscoe 1996; Jones 1996) mention the importance of punctuation and explore this problem from the viewpoint of NLP.

At the beginning of this study, we did not realize that punctuation pattern affects readability. When we asked the intermediate non-native speakers to assess the generation result such as Text (3), almost all human subjects pointed out that they did not like the text because it had too many commas. Some subjects suggested that if substituted a full stop for the comma at the end of the second clause, the generated text would be easy to read. At that time, we realized that not only the between-text-span punctuation in the local structure but also the punctuations in the linear structure affect the readability.

3. If a liquid metal is cooled quickly, the crystals in it have little time to grow, but if it is cooled slowly, large crystals can grow.

Since comma and full stop are more often used than any other punctuation marks, we analysed the first 900 sentences within TANN to find the punctuation patterns of these sentences. Moreover, comma is used to delimit a sentence into more than one part, which may be words, phrases, or clauses. For example, in Text (4) and Text (5), comma delimits words and phrases. However, in Text (6), comma delimits clauses. In this study, we only consider the comma which is used to delimit clauses.

4. Bill can speak French, English, Spanish and German. 
5. Rebuilding that school, Mr. Brown predicted, will take another 2 years.

6. Small winds can cause ripples, while strong winds create large hurricane waves.

Table 4 shows the investigation results of punctuation patterns. It is obvious that Pattern 1, 2 and 3 often occur, while Pattern 4, 5 and 6 seldom occur. So we can infer that Pattern 4, 5 and 6 can not improve the readability of texts.

We divided the punctuation pattern into three states:

(1) Good pattern. It refers to a linear structure which contains Pattern 1, 2, or 3 . For example, "The coal burned in power stations contains sulphur as an impurity. When the coal is burnt, the sulphur is burnt too.". Its punctuation pattern can be represented as

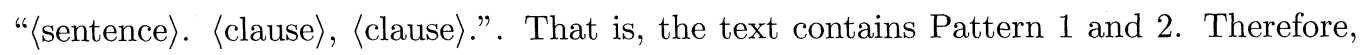
this is the "good pattern".

(2) Normal pattern. It refers to a linear structure which contains Pattern 4, 5, or 6, besides (or without) Pattern 1, 2 or 3. For example, "The shape of metals can be changed because the layers of atoms can slide past or over each other. When they do this, some bonds are broken, but an equal number are made.". Its punctuation pattern can be represented as " $\langle$ clause $\rangle\langle$ clause $\rangle .\langle$ clause $\rangle,\langle$ clause $\rangle,\langle$ clause $\rangle . "$. That is, the text contains Pattern 3 and 4. Therefore, this is a "normal pattern".

(3) Bad pattern. It refers to a linear structure which contains none of the patterns shown in Table 4, for example, the punctuation pattern of Text (3).

Heuristic 4 Preferences among states for punctuation pattern: good pattern $>$ normal pattern $>$ bad pattern

Table 4 Punctuation patterns within TANN

\begin{tabular}{|c|l|c|}
\hline & \multicolumn{1}{|c|}{ Patterns } & Frequency \\
\hline \hline 1 & $\langle$ sentence $\rangle$. & 653 \\
2 & $\langle$ clause $\rangle,\langle$ clause $\rangle$. & 143 \\
3 & $\langle$ clause $\rangle\langle$ clause $\rangle$. & 73 \\
4 & $\langle$ clause $\rangle,\langle$ clause $\rangle,\langle$ clause $\rangle$. & 18 \\
5 & $\langle$ clause $\rangle,\langle$ clause $\rangle\langle$ clause $\rangle$. & 10 \\
\multirow{2}{*}{6} & $\langle$ clause $\rangle\langle$ clause $\rangle,\langle$ clause $\rangle$. & 3 \\
\hline \hline \multicolumn{2}{|c|}{ Total } & 900 \\
\hline
\end{tabular}




\section{Text generation using a GA}

The task of microplanner is to decide how the sentences from the tree will be ordered and punctuated, and selects one text whose readability is appropriate for intermediate non-native users. For an RST-tree, such as the one shown in Fig. 1, the number of possible combinations of span order and between-text-span punctuation is very huge and the search space is not perfectly smooth. Therefore, the task of generation does not necessarily require a global optimum. A combination that could be understood by non-native users without difficulties would be enough. Based on this consideration, we think a Genetic Algorithm is suitable for solving such a problem.

Fig. 3 illustrates the flow diagram of Simple Genetic Algorithm. It shows that the GA analyses the input RST-tree and then generates an optimized text. In this section, we focus on introducing chromosome decoding (Section 4.1) and fitness evaluation (Section 4.2).

\subsection{Using 3 genes to represent the local structure of a candidate gen- eration result}

In a local structure, the position of its child nodes has two choices: "nucleus - satellite" and "satellite - nucleus". The between-text-span punctuation has three choices: space (i.e. no punctuation), comma, and full stop. Therefore, the position of child nodes and between-text-span punctuation have 6 combinations.

For each sub-tree, we use 3 genes to represent the local structure of a candidate generation result (Fig. 4). Each gene has two scores: 0 and 1. The first gene represents the nucleus position: "0" means the nucleus is placed in the first span, i.e. "nucleus - satellite"; "1" means

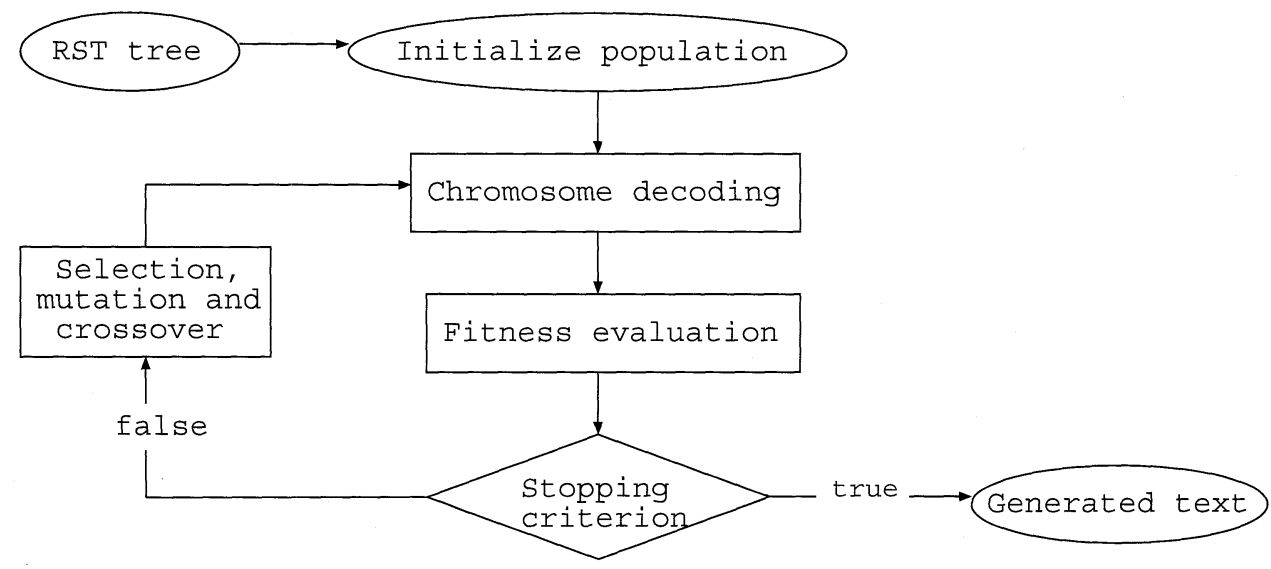

Fig. 3 Flow diagram of Simple Genetic Algorithm applied to the microplanner 


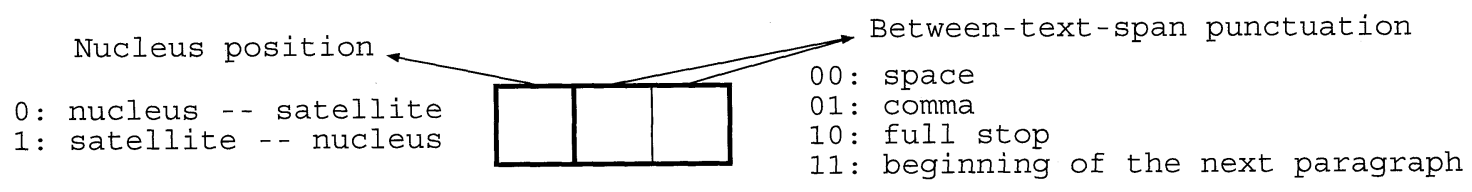

Fig. 4 Using 3 genes to represent the local structure of a candidate generation result

Table 5 Genes and their representations

\begin{tabular}{|c|c|l|}
\hline & Genes & \multicolumn{1}{|c|}{ Representations } \\
\hline \hline 1 & 000 & nucleus + space + satellite \\
\hline 2 & 001 & nucleus + comma + satellite \\
\hline 3 & 010 & nucleus + full stop + satellite \\
\hline 4 & 011 & nucleus + beginning of the next paragraph + satellite \\
\hline 5 & 100 & satellite + space + nucleus \\
\hline 6 & 101 & satellite + comma + nucleus \\
\hline 7 & 110 & satellite + full stop + nucleus \\
\hline 8 & 111 & satellite + beginning of the next paragraph + nucleus \\
\hline
\end{tabular}

the nucleus is placed in the second span, i.e. "satellite - nucleus". The second and the third genes represent the between-text-span punctuation: "00" means space; "01" means comma; " 10 " means full stop; "11" means beginning of the next paragraph. Since we do not consider the problem of generating a text with more than one paragraph, "11" is not desirable, so its score is negative in the experiments. Table 5 shows the eight combinations of the three genes and their representations.

\subsection{Evaluating a candidate generation result}

A key requirement of the GA approach is the ability to evaluate the quality of a possible solution. We assign one candidate generation result of a sub-tree a score which is the sum of scores of the preferences for the four factors. For a whole RST-tree, the fitness of the candidate generation result is the sum of the scores of all sub-trees it has. We use the RST-tree shown in Fig. 1 to illustrate how the microplanner works.

In order to justify our opinion that it is the preferences among the factors that determine the readability of the texts on discourse level, we fed the four heuristics mentioned in Section 3 into a constraint-based program. The program then randomly selects a number for each preference of the factors according to the heuristics. Table 6 shows a value generated from the program. 
Table 6 A value used in the experiment

\begin{tabular}{|c|l|c|}
\hline \multicolumn{1}{|c|}{ Factors } & \multicolumn{1}{|c|}{ Preferences } & Value \\
\hline \hline Nucleus position & good position & 15 \\
& normal position & 1 \\
& bad position & -3 \\
\hline \multirow{3}{*}{ Between-text-span punctuation } & good punctuation & 6 \\
& bormal punctuation & 2 \\
& good punctuation & -9 \\
\hline EDMs & normal EDMs & 5 \\
& bad EDMs & -2 \\
\hline Punctuation pattern & good pattern & 14 \\
& normal pattern & 11 \\
& bad pattern & -7 \\
\hline
\end{tabular}

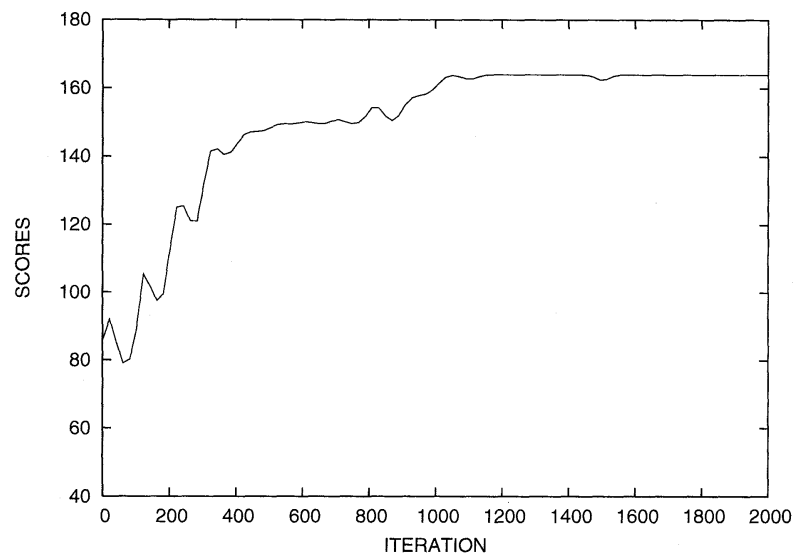

Fig. 5 Scores of the best texts for 2000 iterations

The negative scores are given to unfavourable preferences and positive scores to favourable ones. Using this value, we ran the GA on the RST-tree shown in Fig. 1.

Fig. 5 shows the scores of the best texts (2000 iterations). We can see that the scores keep on improving and gets stable at around 1600 iterations. At this moment, the best text (see Fig. 2 ) is scored at 164 . The scoring is summarised in Table 7. In the first column, the root, gene representation and local structure of each sub-tree are shown. In the third column, N, B, E, P represent the value of nucleus position, between-text-span punctuation, EDMs and punctuation 
Table 7 Summary of the scores of the best text

\begin{tabular}{|c|c|c|}
\hline Sub-tree & Linear structure & Scores \\
\hline $\begin{array}{l}\text { Root: Node } 3 \\
\text { Genes: } 101 \\
\text { Local structure: } \\
\text { satellite + comma + nucleus }\end{array}$ & $\begin{array}{l}\text { If you do things at the wrong time of } \\
\text { year, the results will not be so good. }\end{array}$ & $\begin{array}{c}40 \\
(\mathrm{~N}: 15, \mathrm{~B}: 6 \\
\mathrm{E}: 5, \mathrm{P}: 14)\end{array}$ \\
\hline $\begin{array}{l}\text { Root: Node } 4 \\
\text { Genes: } 101 \\
\text { Local structure: } \\
\text { satellite + comma + nucleus }\end{array}$ & $\begin{array}{l}\text { If you grow seeds in the fields at the } \\
\text { correct time of year, the results will } \\
\text { be better. }\end{array}$ & $\begin{array}{l}\quad 40 \\
(\mathrm{~N}: 15, \mathrm{~B}: 6 \\
\mathrm{E}: 5, \mathrm{P}: 14)\end{array}$ \\
\hline $\begin{array}{l}\text { Root: Node } 2 \\
\text { Genes: } 110 \\
\text { Local structure: } \\
\text { satellite + full stop + nucleus }\end{array}$ & $\begin{array}{l}\text { If you grow seeds in the fields at the } \\
\text { correct time of year, the results will } \\
\text { be better. But if you do things at the } \\
\text { wrong time of year, the results will } \\
\text { not be so good. }\end{array}$ & $\begin{array}{l}42 \\
(\mathrm{~N}: 15, \mathrm{~B}: 6 \\
\mathrm{E}: 7, \mathrm{P}: 14)\end{array}$ \\
\hline $\begin{array}{l}\text { Root: Node } 0 \\
\text { Genes: } 010 \\
\text { Local structure: } \\
\text { nucleus + full stop + satellite }\end{array}$ & $\begin{array}{l}\text { Plant at the right time of year. If } \\
\text { you grow seeds in the fields at the } \\
\text { correct time of year, the results will } \\
\text { be better. But if you do things at the } \\
\text { wrong time of year, the results will } \\
\text { not be so good. }\end{array}$ & $\begin{array}{l}\quad 42 \\
(\mathrm{~N}: 15, \mathrm{~B}: 6 \\
\mathrm{E}: 7, \mathrm{P}: 14)\end{array}$ \\
\hline & Fitness & 164 \\
\hline
\end{tabular}

pattern.

\section{Evaluation}

Since evaluation is an important issue in NLG, researchers have been trying to find valid and widely accepted methods. In (Coch 1996), the author used a black-box methodology to assess three techniques for producing multisentential texts. The research of (Yeh and Mellish 1997) compared human-created results and computer-generated results. Moreover, in (Williams, Reiter, and Osman 2003; Williams and Reiter 2004), the authors evaluated readability of the output texts by measuring reading speed and reading errors. However, we do not think this 
method is appropriate for our study, because many intermediate (even advanced) non-native speakers do not know how to pronounce English words. In China and Japan, the reality is many graduate school students can not hold a conversation with a person in English even if they can write technical papers in English without difficulties. Therefore, we adopt the following two evaluation methods: evaluating the reliability of the SILK system by analysing how often it re-generates corpus texts, and judging readability by human subjects.

\subsection{Evaluating the reliability of the SILK system}

In order to evaluate the reliability of the SILK system, we analyse how often it re-generates corpus texts. Generally, the higher the frequency of re-generating corpus texts is, the more reliable a text generation system is.

We selected 30 texts from corpus TANN, and asked two trained coders to annotate the structure of these texts by RST. Then we compared the annotation results of the two coders. Of the 30 texts, the annotation results of 22 texts were consistent with each other. Therefore, we used the 22 texts in the experiments.

Using the value shown in Table 6, we ran the GA for 5000 iterations on the RST-tree of each text for 100 times. For each tree structure, we counted how often the system re-generates TANN

Table 8 Frequency of re-generating corpus texts

\begin{tabular}{|c|c|c||c|c|c|}
\hline Text & $\begin{array}{c}\text { Number of } \\
\text { discourse } \\
\text { relations }\end{array}$ & Frequency & Text & $\begin{array}{c}\text { Number of } \\
\text { discourse } \\
\text { relations }\end{array}$ & Frequency \\
\hline \hline 1 & 2 & 74 & 12 & 4 & 47 \\
\hline 2 & 2 & 86 & 13 & 4 & 42 \\
\hline 3 & 2 & 79 & 14 & 4 & 50 \\
\hline 4 & 2 & 83 & 15 & 5 & 40 \\
\hline 5 & 2 & 81 & 16 & 5 & 33 \\
\hline 6 & 3 & 67 & 17 & 5 & 36 \\
\hline 7 & 3 & 55 & 18 & 6 & 30 \\
\hline 8 & 3 & 58 & 19 & 6 & 25 \\
\hline 9 & 3 & 63 & 20 & 7 & 28 \\
\hline 10 & 3 & 60 & 21 & 7 & 22 \\
\hline 11 & 4 & 52 & 22 & 10 & 11 \\
\hline
\end{tabular}


text (i.e. how often the output text is same as the original TANN text). The experimental results are shown in Table 8. In the table, the second column and the fifth column represent the number of discourse relations the texts contain. The third column and the sixth column represent the frequency of re-generating corpus texts.

We can see that the frequency of re-generating corpus texts is dependent on the number of discourse relations the texts contain, i.e. the more relations a text contains, the lower the frequency of re-generating corpus text is. In Table 8, for the texts containing 2 relations, the frequency ranges from 74 to 86 , but for the text containing 10 relations, the frequency is 11 .

We take Text 11 as an example to illustrate the experiment results. Fig. 1 shows the RSTtree of Text 11, and the text shown in Fig. 2 is same as its original TANN text. We found that the frequency of re-generating corpus texts is 52 after we ran the GA for 100 times. There are another 41 output texts can be understood, though they are not consistent with the original TANN text. For example, "Plant at the right time of year. The results will be better if you grow seeds in the fields at the correct time of year. But if you do things at the wrong time of year, the results will not be so good." and "Plant at the right time of year. If you grow seeds in the fields at the correct time of year, the results will be better. But the results will not be so good if you do things at the wrong time of year." Moreover, there are only 7 output texts that can not be completely understood. For example, "Plant at the right time of year if you grow seeds in the fields at the correct time of year, the results will be better. But if you do things at the wrong time of year. The results will not be so good." and "The results will be better. If you grow seeds in the fields at the correct time of year, but the results will not be so good if you do things at the wrong time of year. Plant at the right time of year.".

Actually, within TANN, the average sentences that per paragraph contains is 3.1 , which means that intermediate non-native audience prefers shorter texts. Therefore, we can say that so long as a text does not contain too many discourse relations (e.g. less than 5 relations), most of the generated texts can be understood. The experiment results show that the SILK is a high-reliability text generation system.

\subsection{Judging readability by human subjects}

To prove the GA method could simplify the English text on discourse level, we designed a questionnaire and asked human subjects to compare the RST-DTC ${ }^{5}$ texts to the output texts produced by SILK using the tree structures of the RST-DTC texts.

\footnotetext{
${ }^{5}$ RST-DTC is the largest publicly available discourse-annotated corpus using the framework of RST. It can be obtained from the Linguistic Data Consortium (USA). The corpus contains articles of the Wall Street Journal.
} 
We selected 20 texts from RST-DTC. Using the value shown in Table 6, we ran the GA for 5000 iterations on the tree structure of each RST-DTC text for five times, and chose the best result (i.e. the one with the highest score). In the questionnaire, we place the best text (i.e. SILK text) and its original RST-DTC text on the same page so that the subjects can compare them easily. And then we asked the subjects to choose a text they prefer.

52 intermediate non-native subjects took part in the experiment. In this study, a person whose school background is lower than university is regarded to be an intermediate non-native speaker. Of the subjects, 27 are high school students, 9 are junior college students, 16 graduated from junior college. Moreover, 23 are male, 29 are female. The age of the subjects ranges from 17 to 62 ; the mean is 31 . We contacted with the human subjects by Internet and asked them to finish the questionnaire in their spare time.

Table 9 shows the results of the questionnaire. The second column and the fifth column represent the percentage of the subjects who prefer the SILK texts. The third column and the sixth column represent the percentage of the subjects who prefer the RST-DTC texts. We can see that most of the subjects prefer SILK texts (except the one in Question 16). This shows that the GA method can simplify English texts on discourse level and the generated texts are easy to read for intermediate non-native speakers.

Further analysis shows that the RST-tree used in Question 16 is a complicated one, which contains 11 discourse relations. The SILK text in Question 16 begins with "For example, but

Table 9 The questionnaire results

\begin{tabular}{|c|c|c|c|c|c|}
\hline \multirow{2}{*}{ Question } & \multicolumn{2}{|c|}{ Percentage } & \multirow{2}{*}{ Question } & \multicolumn{2}{|c|}{ Percentage } \\
\cline { 2 - 3 } & SILK text & RST-DTC text & & SILK text & RST-DTC text \\
\hline \hline 1 & 88 & 12 & 11 & 83 & 17 \\
\hline 2 & 81 & 19 & 12 & 94 & 6 \\
\hline 3 & 96 & 4 & 13 & 81 & 19 \\
\hline 4 & 90 & 10 & 14 & 92 & 8 \\
\hline 5 & 83 & 17 & 15 & 94 & 6 \\
\hline 6 & 90 & 10 & 16 & 6 & 94 \\
\hline 7 & 88 & 12 & 17 & 96 & 4 \\
\hline 8 & 92 & 8 & 18 & 88 & 12 \\
\hline 9 & 85 & 15 & 19 & 81 & 19 \\
\hline 10 & 94 & 6 & 20 & 88 & 12 \\
\hline
\end{tabular}


its greatest drawback may be its 3-inch thickness, big enough for one consultant to describe it as "clunky."". It is obvious that "For example, but" are bad EDMs, which are unfavourable preference. It is the beginning of the text that makes the human subjects confused. Moreover, in the experiment, we only ran the GA on the RST-tree of each text for five times. For a text containing 11 relations, since the number of possible combinations is huge (i.e. $2^{33}$ ), we can not guarantee the best one of the five generation results is appropriate for intermediate non-native audience. Actually, we will not generate longer texts for intermediate non-native users, so the generation result such as the SILK text in Question 16 can be avoided.

\section{Related work}

The aim of this study is to simplify English text for intermediate non-native speakers. In NLG, simplification of English document is a relatively new research field. The first study for ease of comprehension in NLG output was (Scott and Souza 1990). The authors point out that generating discourse markers whenever possible could make a text easier to comprehend. Moreover, several other methods were used to simplify texts. The study of (Devlin and Tait 1998) substituted common words for uncommon words. In (Chandrasekar and Srinivas 1997), the authors reduced multiple-clause sentences to single-clause sentences. In (Devlin, Canning, Tait, Carrol, Minnen, and Pearce 2000), the authors simplified newspaper articles for aphasic readers. Their research focused on the simplification of syntactic structures and lexical simplification.

Until now, there exists a research focusing on simplification of English texts on discourse level. The GIRL system (Williams 2004) can generate texts for native speakers who have poor reading skills due to a number of reasons, such as missed school, poor eyesight, short-term memory, etc. Williams (2004) claims that length and ordering of the text spans, position of discourse marker, between-text-span punctuation and selection of discourse marker affect the readability on discourse level. In GIRL, the inputs to the microplanner are a model of a user's reading ability and a discourse plan containing the discourse relation tree. The user model is built from user's answers to up to ninety questions from a literacy test. Based on the user models, GIRL can adapt the generated texts to the reading level of individual users.

\section{Conclusion}

In this study, we applied GA to develop the microplanner of the SILK system which can generate texts appropriate for intermediate non-native speakers on discourse level. We think 
that four factors affect the readability, i.e. nucleus position, between-text-span punctuation, embedded discourse markers and punctuation pattern. We claim that it is the preferences among these factors that determine the readability. We adopt two methods to evaluate the system: one is evaluating the reliability of the SILK system by analysing how often it re-generates corpus texts, another is judging readability by human subjects. The evaluation results show that the system is reliable and the generated texts are appropriate for intermediate non-native users.

The GA method introduced in this paper could be extended in generating other types of texts, for example, world affairs, arts, etc. Moreover, the architecture based on the Genetic Algorithm can also be applied to text generation for users with different levels, such as children and middle school students. To realize these aims, it is necessary to do more research to explore the influence of the factors mentioned above on the reading ability of the users.

This study is the first stage of developing the SILK system. Future work will focus on simplification of syntactic structures and lexical simplification. Our aim is to make the SILK system generate texts appropriate for intermediate non-native speakers not only on discourse level but also on sentence level.

\section{Acknowledgment}

I would like to thank Ehud Reiter, Sandra Williams and an anonymous JNLP reviewer for their helpful comments on this paper.

\section{Appendix}

\section{The nucleus position}

We used machine learning program C4.5 (Eugenio et al. 1997) to induce the classification model of the nucleus position for each relation used in this study. The examples of each relation were obtained from corpus TANN. Here, we use "reason" relation signaled by because to illustrate the method of investigating the nucleus position.

We selected the first 120 examples of "reason" relation signaled by because. Before the exper-

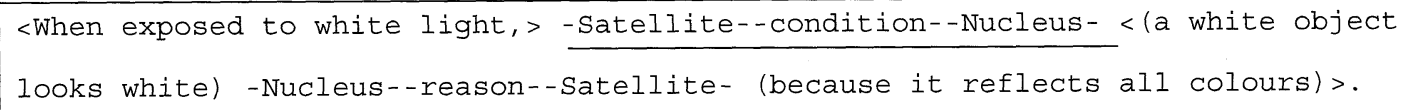

Fig. 6 An annotation result 
iments, two trained coders took part in annotating the examples. An annotation result is shown in Fig. 6, in which the portion annotated in round brackets is called embedded structure and the one annotated in angle brackets is called whole structure. We can see that the embedded structure is contained in the nucleus of "condition" relation signaled by when, i.e. the whole structure includes the embedded structure. Moreover, the embedded structure contains the "reason" relation signaled by because.

In the experiments, each data point in our dataset is characterized by 18 features. These features are divided into the following two groups.

1. Embedded structure features

(1) Mt. Tense of the main clause: past, present, future.

(2) Mv. Voice of the main clause: active, passive.

(3) Mg. Length of the main clause (in words): integer.

(4) Ms. Structure of the main clause: simple, other.

(5) Mi. Information contained in the main clause: new, old.

(6) St. Tense of the subordinate clause: past, present, future.

(7) Sv. Voice of the subordinate clause: active, passive.

(8) Sg. Length of the subordinate clause (in words): integer.

(9) Ss. Structure of the subordinate clause: simple, other.

(10) Si. Information contained in the subordinate clause: new, old.

2. Whole structure features

(1) R. Discourse relation: background, condition, contrast, elaboration, evaluation, example, list, purpose, reason, restatement, summary, time.

(2) X. Whether the discourse relation is signaled by discourse marker: yes, no.

(3) E. Role of the embedded structure: nucleus, satellite.

(4) P. Position of the embedded structure: first span, second span.

(5) Eg. Length of the span containing the embedded structure: integer.

(6) Es. Structure of the span containing the embedded structure: complex sentence, other.

(7) Og. Length of the span not containing the embedded structure: integer.

(8) Os. Structure of the span not containing the embedded structure: simple sentence, other.

We used the 120 annotated examples to induce the classification models of the nucleus position of "reason" relation. Within the 120 examples, $70.8 \%$ (85/120) of nuclei occur in the first span. Therefore, the error rate of the baseline model is $29.2 \%$. We did 38 experiments using different models represented by subsets of the 18 features. The first 18 experiments used the models 
represented by individual features, corresponding to each feature mentioned above. Another 20 experiments used the models represented by the combinations of these features. We identify the best learned model(s) by comparing it (their) error rate(s) with the error rates of the other learned models and with the error rate of the baseline model. That is, if the upper bound of the $95 \%$ confidence interval for error rate $\varepsilon 1$ is lower than the lower bound of the $95 \%$ confidence interval for error rate $\varepsilon 2$, then the difference between $\varepsilon 1$ and $\varepsilon 2$ is considered to be significant.

Table 10 shows the error rates of four classification models which perform better than the baseline model. Of these models, the one learned from feature $\mathrm{R}$ is the best model because it performs better than the three others. The model says that if the discourse relation of the whole structure is "contrast", "example" or "reason", then in the embedded structure, the nucleus of

Table 10 95\%-confidence intervals for the error rates for four classification models of "reason" relation signaled by because

\begin{tabular}{|c|c|c|c|c|}
\hline & \multicolumn{4}{|c|}{ Models } \\
\hline $\mathrm{Mt}$ & & & $\mathrm{x}$ & $\mathrm{x}$ \\
\hline $\mathrm{Mv}$ & & & $\mathrm{x}$ & $\mathrm{x}$ \\
\hline $\mathrm{Mg}$ & & & $x$ & $x$ \\
\hline Ms & & & $\mathrm{x}$ & $\mathrm{x}$ \\
\hline $\mathrm{Mi}$ & & & $\mathrm{x}$ & \\
\hline St & & & $\mathrm{x}$ & $\mathrm{x}$ \\
\hline $\mathrm{Sv}$ & & & $\mathrm{x}$ & $\mathrm{x}$ \\
\hline $\mathrm{Sg}$ & & & $\mathrm{x}$ & $\mathrm{x}$ \\
\hline $\mathrm{Ss}$ & & & $x$ & $x$ \\
\hline $\mathrm{Si}$ & & & $\mathrm{x}$ & \\
\hline $\mathrm{R}$ & $\mathrm{x}$ & $\mathrm{x}$ & $\mathrm{x}$ & $\mathrm{x}$ \\
\hline $\mathrm{X}$ & & $\mathrm{x}$ & $\mathrm{x}$ & $\mathrm{x}$ \\
\hline $\mathrm{E}$ & & $\mathrm{x}$ & $\mathrm{x}$ & $\mathrm{x}$ \\
\hline $\mathrm{P}$ & & $\mathrm{x}$ & $\mathrm{x}$ & $\mathrm{x}$ \\
\hline $\mathrm{Eg}$ & & $x$ & $\mathrm{x}$ & $\mathrm{x}$ \\
\hline Es & & $\mathrm{x}$ & $\mathrm{x}$ & $\mathrm{x}$ \\
\hline $\mathrm{Og}$ & & $\mathrm{x}$ & $\mathrm{x}$ & $\mathrm{x}$ \\
\hline Os & & $\mathrm{x}$ & $\mathrm{x}$ & $\mathrm{x}$ \\
\hline Results & $15.8 \pm 2.66$ & $24.2 \pm 2.62$ & $23.3 \pm 3.80$ & $22.5 \pm 4.01$ \\
\hline
\end{tabular}


"reason" relation signaled by because occurs in the second span; otherwise occurs in the first span.

\section{Reference}

Bateman, J. (1997). "Enabling technology for multilingual natural language generation: The KPML development environment." Natural Language Engineering, 3 (1), pp. 15-55.

Biber, D., Johansson, S., Leech, G., Conrad, S., and Finegan, E. (1999). Longman grammar of spoken and written English. Pearson Education Limited, England.

Briscoe, T. (1996). "The Syntax and Semantics of Punctuation and its Use in Interpretation." In Proc. of the Association for Computational Linguistics Workshop on Punctuation, pp. 1-7.

Chandrasekar, R. and Srinivas, B. (1997). "Automatic induction of rules for text simplification." Knowledge-Based System, 10 (3), pp. 183-190.

Coch, H. (1996). "Evaluating and Comparing Three Text-production Techniques." In Proc. of the 16th International Conference on Computational Linguistics, pp. 249-254.

Dale, R. (1991). "Exploring the Role of Punctuation in the Signaling of Discourse Structure." In Proc. of Workshop on Text Representation and Domain Modeling: Ideas from Linguistics and $A I$, pp. 110-120.

Devlin, S., Canning, Y., Tait, J., Carrol, J., Minnen, G., and Pearce, D. (2000). "Making accessible international communication for people with language comprehension difficulties." In Proc. of The 7th International Conference on Computers Helping People with Special Needs, pp. 135-142.

Devlin, S. and Tait, J. (1998). "The use of a psycholinguistic database in the simplification of text for aphasic readers." In Nerbonne, J. (Ed.), Linguistic Databases, pp. 161-173. CSLI, New York.

Elhadad, M. and McKeown, K. (1990). "Generating connectives." In Proc. of the Thirteenth International Conference on Computational Linguistics, pp. 97-101.

Eugenio, B., Moore, J., and Paolucci, M. (1997). "Learning Features that Predict Cue Usage." In Proc. of the 35th Annual Meeting of the Association for Computational Linguistics, pp. $80-87$.

Goldberg, E., Driedger, N., and Kittredge, R. (1994). "Using natural language processing to produce weather forecasts." IEEE Expert, 9 (2), pp. 45-53.

Hirano, H. (2003). Genetic Algorithms and Genetic Programming. Personal Media, Tokyo.

Hovy, E. and Arens, Y. (1991). "Automatic Generation of Formatted Text." In Proc. of AAAI-91, 
pp. 92-97.

Jones, B. (1996). "Towards a Syntactic Account of Punctuation." In Proc. of the 16th International Conference on Computational Linguistics, pp. 604-609.

Mann, W. and Thompson, S. (1988). "Rhetorical structure theory: Toward a functional theory of text organization." Text, 8 (3), pp. 243-281.

McKeown, K. (1985). Text Generation. Cambridge University Press.

Moser, M. and Moore, J. (1995). "Investigating cue selection and placement in tutorial discourse." In Proc. of the 33rd Annual Meeting of the Association for Computational Linguistics, pp. 130-135.

Power, R., Doran, C., and Scott, D. (1999). "Generating embedded discourse markers from rhetorical structure." In Proc. of the 7th European Workshop for Natural Language Generation, pp. 33-38.

Scott, D. and Souza, C. (1990). "Getting the Message Across in RST-based Text Generation." In Dale, R., Mellish, C., and M., Z. (Eds.), Current Research in Natural Language Generation, pp. 47-73. Academic Press.

White, M. (1995). "Presenting Punctuation." In Proc. of the Fifth European Workshop on Natural Language Generation, pp. 107-125.

Williams, S. (2003). "Language choice models for microplanning and readability." In Proc. of the Student Workshop of the Human Language Technology and North American Chapter of the Association for Computational Linguistics Conference, pp. 13-18.

Williams, S. (2004). Natural language generation (NLG) of discourse relations for different reading levels. Ph.D. thesis, University of Aberdeen.

Williams, S. and Reiter, E. (2004). "Reading errors made skilled and unskilled readers: evaluating a system that generates reports for people with poor literacy." Tech. rep., University of Aberdeen Department of Computer Science Technical Report AUCS/TR0407.

Williams, S., Reiter, E., and Osman, L. (2003). "Experiments with discourse-level choices and readability." In Proc. of the 9th European Workshop on Natural Language Generation, pp. 127-134.

Yeh, C. and Mellish, C. (1997). "An Empirical Study on the Generation of Anaphora in Chinese." Computational Linguistics, 23 (1), pp. 171-190.

Xinyu Deng: Xinyu Deng received her B.E. and M.A. from Harbin Institute of Technology, China. From 2001 to 2003 she worked for R\&D center of Toshiba, 
Tokyo, Japan. She completed her Ph.D. course of Kyoto University in 2006. She is currently doing research at Kyoto University. Her research interests include natural language processing, machine translation and corpus linguistics.

Jun-ichi Nakamura: (1956 - 2001) Jun-ichi Nakamura received his BSc, MSc and DSc in electrical engineering from Kyoto University in 1979, 1981, and 1989. From 1997 to 2001 he was a professor in Kyoto University.

(Received July 1, 2006)

(Revised September 1, 2006)

(Accepted October 6, 2006) 